\title{
Specific $D$-Admissibility and Design Issues for Uncertain Descriptor Systems with Parametric Uncertainty in the Derivative Matrix
}

\author{
Chih-Peng Huang \\ Department of Computer Science, University of Taipei, Taipei 100, Taiwan \\ Correspondence should be addressed to Chih-Peng Huang; cphuang@utaipei.edu.tw
}

Received 30 October 2015; Revised 8 January 2016; Accepted 10 January 2016

Academic Editor: Yan-Jun Liu

Copyright (C) 2016 Chih-Peng Huang. This is an open access article distributed under the Creative Commons Attribution License, which permits unrestricted use, distribution, and reproduction in any medium, provided the original work is properly cited.

\begin{abstract}
Stability analysis issues and controller synthesis for descriptor systems with parametric uncertainty in the derivative matrix are discussed in this paper. The proposed descriptor system can extend the system's modeling extent of physical and engineering systems from the traditional state-space model. First, based on the extended $D$-stability definitions for the descriptor model, necessary and sufficient admissibility and $D$-admissibility conditions for the unforced nominal descriptor system are derived and formulated by compact forms with strict linear matrix inequality (LMI) manner. In contrast, existing results need to involve nonstrict LMIs, which cannot be evaluated by current LMI solvers and need some extra treatments. Deducing from the obtained distinct results, the roust admissibility and $D$-admissibility of the descriptor system with uncertainties in both the derivative matrix and the system's matrices thus can be coped. Furthermore, by involving a proportional and derivative state feedback (PDSF) control law, we further address the controller design for the resulting closed-loop systems. Since all the proposed criteria are explicitly expressed in terms of the strict LMIs, we can use applicable LMI solvers for evaluating the feasible solutions. Finally, the efficiency and practicability of the proposed approach are demonstrated by two illustrative examples.
\end{abstract}

\section{Introduction}

For capably integrating dynamic behaviors and algebraic dependent constraints into a single system's model, descriptor systems have revealed more applicable fields and research topics than the conventional state-space models especially, such as electrical networks, power systems, robotic systems, chemical processes, and social economic system [1-4]. They also become a powerful technique, named by a descriptor system approach, to solve many concerned control problems via specific augmented forms $[5,6]$. Descriptor systems also are named as generalized state-space systems, differentialalgebraic systems, singular systems, implicit systems, or semistate systems. But, the arising stability issues are more intractable than the conventional state-space ones. Besides the fundamental stability, we need extra treatment to assure the regularity and impulse immunity simultaneously (see, e.g., [7-13], and the references therein).
Furthermore, considering the tolerable disturbance of realistic systems, mathematical modeling needs to embrace parameter's uncertainties against rounding errors, varying operation circumstance, components' aging, and so on. Some of them also can be transferred as nonlinear systems with dead zone input and the corresponding stability issues then can be efficiently treated by adaptive control strategy [14-16]. For considering descriptor systems with uncertainties, even if the nominal descriptor system is admissible, the stability, regularity, or impulse immunity can easily be destroyed by the arising uncontrollable uncertainties. Some works [8, 17-19] dealt with the robust admissibility and robust stabilization for the uncertain descriptor systems with a constant derivative matrix E. But, for mathematical modeling of a physical system, if there exists parametric perturbation within its inner structures or behaviors, they should be meaningfully represented as parametric uncertainties directly into both the system matrix and the derivative matrix. Thus, few 
works [20, 21] have set about addressing the uncertain descriptor systems with the perturbed derivative matrices, where the presented uncertain derivative matrices had to meet some specific forms. Nevertheless, for considering the system's performance, the system's transient behaviors can be metricized by decay rate, damping, settle time, and so on. These characterized performance indexes mainly are dominated by the poles' clustering location of the statespace model. Thus, for the regular state-space model, poles' locations in various geometric regions, for example, shift half planes, vertical/horizontal strips, sectors, parabolic regions, and any intersection thereof, were deeply discussed in many works [22-28]. And the descriptor systems with specific poles' location also have been discussed very recently [2931]. However, the stability region analysis for the uncertain descriptor systems with the uncertain derivative matrix has drawn little attention in the past.

Furthermore, if the derivative of the state $x(t)$ (or the output $y(t))$ is accessible during the control process, a proportional and derivative state feedback (PDSF) (or proportional and derivative output feedback (PDOF)) controller can be equipped to stabilize the closed-loop descriptor systems [32-37]. But, some results are based on the matrix decomposition, and they cannot be extended to embrace uncertain parameters. To date, robust normalization and stabilization for uncertain descriptor systems were addressed [36]. According to an augmented form approach associated with a PDSF (or (PDOF)) controller, they presented some design criteria for the resulting closed-loop descriptor system with norm-bounded uncertainties in the derivative matrix and the system's matrices simultaneously.

In this study, we investigate the robust $D$-admissibility and the controller design for the descriptor system with the existing uncertainties in both the derivative matrix and the system matrices simultaneously. Compact admissibility and $D$-admissibility conditions for the nominal descriptor system, which can be directly expressed by one strict linear matrix inequality (LMI), are first proposed. In contrast, some existing results involve additional nonstrict LMIs $E^{T} P=$ $P E^{T} \geq 0$ [38] or $E^{T} Q E \geq 0$ [12] in their results, where they cannot be directly evaluated by current LMI solvers and need some extra treatments [39, 40]. Based on the proposed explicit forms, we thus can treat the admissibility and $D$-admissibility for the system with uncertainties in both the derivative matrix and the system matrix. In addition, a PDSF control law is involved to normalize and stabilize the closed-loop descriptor models. Since all the proposed criteria are explicitly expressed by strict LMIs' forms, we can handily evaluate them via existing LMI tools [40, 41] for verification. The remainder content is organized as follows. The concerned problem and some preliminary results are described in Section 2. The robust admissibility and $D$ admissibility issues are addressed in Section 3. In Section 4, an augmented form approach associated with the PDSF control is further investigated for the controller design. In Section 5, two illustrative examples are given to demonstrate the applicability and validity of the proposed method. Some conclusions are drawn in Section 6.

\section{Problem Formulation and Preliminaries}

Consider an uncertain descriptor system described as

$$
\widetilde{E} \dot{x}(t)=(A+\Delta A) x(t)+(B+\Delta B) u(t)
$$

where $x(\cdot) \in R^{n}$ is the descriptor vector, $u(\cdot) \in R^{p}$ is the control input, and the perturbed derivative matrix $\widetilde{E} \in R^{n \times n}$ may be singular, that is, $\operatorname{rank}(\widetilde{E})=m \leq n$, and belongs to a polytopic set defined as

$$
\Omega_{E} \equiv\left\{\widetilde{E}: \widetilde{E}=\sum_{i}^{r} e_{i} E_{i}, e_{i} \geq 0, \sum_{i=1}^{r} e_{i}=1\right\},
$$

where $E \in R^{n \times n}$ is given a priori. $A$ and $B$ stand for the nominal system matrices with appropriate dimensions and $\Delta A$ and $\Delta B$ represent the parameter uncertainties bounded by

$$
\left[\begin{array}{ll}
\Delta A & \Delta B
\end{array}\right]=M D\left[\begin{array}{ll}
N_{A} & N_{B}
\end{array}\right]
$$

where $M, N_{A}$, and $N_{B}$ are constant matrices with compatible dimensions and $D$ satisfies

$$
D^{T} D \leq I
$$

Remark 1. For system's model (1), the presented uncertainties in the derivative matrix and the system matrices are formulated by difference forms. For mathematical modeling of a physical system, if there exists parametric perturbation within its inner structures or behaviors, the mainly parametric uncertainties usually can be cast into system matrices and the remainder few uncertainties thus are cast into the derivative matrix. So, we can reasonably formulate the system's matrices with whole uncertainties by the norm bound in (3) and the derivative matrix with individual uncertainties by the polytopic form in (2).

Definition 2 (see $[12,42])$. (i) The pair $(E, A)$ is said to be regular if $\operatorname{det}(s E-A)$ is not identically zero.

(ii) The pair $(E, A)$ is said to be impulse free if $\operatorname{deg}[\operatorname{det}(s E-$ A)] $=\operatorname{rank}(E)$.

(iii) The descriptor system $E \dot{x}(t)=A x(t)$ is said to be admissible if it is regular and impulse free, and $\sigma(E, A)$ lie in the open left half plane, where $\sigma(E, A)$ denotes all roots of $\operatorname{det}(s E-A)=0$.

(iv) The descriptor system $E \dot{x}(t)=A x(t)$ is said to be $D$ admissible if it is regular and impulse free and $\sigma(E, A)$ lie in a specific stability region within the open left half plane.

Lemma 3. The pair $(E, A)$ is regular and impulse free if and only if the pair $(E, z(A-a E)), a \in R, z \in C$, with $z \neq 0$, is regular and impulse free. 
Proof. By replacing the variable $s$ of $\operatorname{det}(s E-A)$ with $(s+z a) / z$ in Definition 2, according to items (i) and (ii) the proof can be directly attained.

Lemma 4 (see [43]). Given a symmetric matrix $\Omega$ and matrices $U$ and $V$ of appropriate dimensions, then

$$
\Omega+U D V+V^{T} D^{T} U^{T}<0
$$

for all $D$ satisfying $D D^{T} \leq I$, if and only if there exists a scalar $\alpha>0$ such that

$$
\Omega+\alpha U U^{T}+\alpha^{-1} V^{T} V<0 .
$$

Denote a specific poles' region in Figure 1. The complex plane is separated into two open-half planes $H$ and $\bar{H}$ by the line $L$ that intersects the real axis at $(-\alpha, 0)$ and the imaginary axis at $(0,-j \beta)$ and makes an angle $\theta$ with respect to the positive imaginary axis, where $\theta$ is defined to be positive with a counterclockwise sense and $-\pi<\theta \leq \pi$ [24]. For the regular state-space system $\dot{x}(t)=A x(t)$, a condition of poles' location in the region $H$ is presented in advance.

Lemma 5 (see [24]). All the poles of the linear system $\dot{x}(t)=$ $A x(t)$ lie in the region $H$, if and only if there exists a positive definite Hermitian matrix $P$ such that

$$
\left[e^{-j \theta}(A+\alpha I)\right]^{*} P+P\left[e^{-j \theta}(A+\alpha I)\right]<0
$$

or

$$
\left[e^{-j \theta}(A+j \beta I)\right]^{*} P+P\left[e^{-j \theta}(A+j \beta I)\right]<0,
$$

where $(\cdot)^{*}$ denotes the complex conjugate transpose of the considered matrix.

According to Lemma 5 with a positive definite Hermitian matrix $P$, a symmetric form is presented as follows.

Corollary 6. All the poles of the linear system $\dot{x}(t)=A x(t)$ lie in the region $H$, if and only if there exists a positive definite Hermitian matrix $P$ such that

$$
\left[e^{-j \theta}(A+\alpha I)\right] P+P\left[e^{-j \theta}(A+\alpha I)\right]^{*}<0
$$

or

$$
\left[e^{-j \theta}(A+j \beta I)\right] P+P\left[e^{-j \theta}(A+j \beta I)\right]^{*}<0 .
$$

\section{Admissible and D-Admissible Analysis}

For the nominal descriptor system $E \dot{x}(t)=A x(t)$, we derive an equivalent admissibility criterion in the following.

Lemma 7 (see [44]). The nominal system $E \dot{x}(t)=A x(t)$ is admissible if and only if there exist a positive definite matrix $P$ and a matrix $Q$ with appropriate dimensions such that

$$
A^{T}(P E-S Q)+(P E-S Q)^{T} A<0,
$$

where matrix $S \in R^{n \times n-m}$ is of full-column rank and satisfies $E^{T} S=0$.

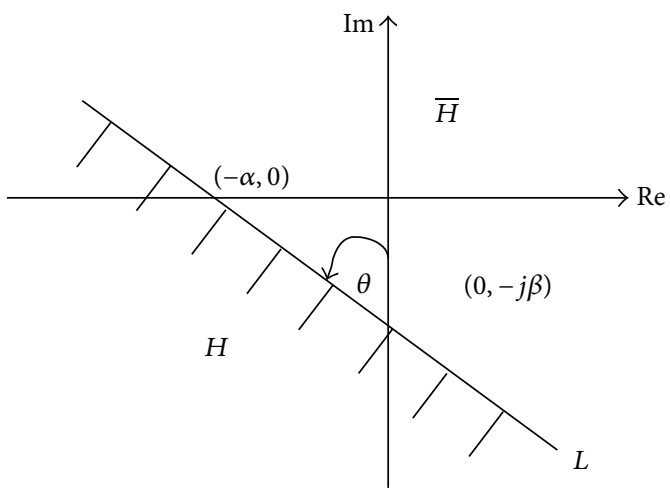

FIgURE 1: An extended pole region [24].

Following the same line of Lemma 7, a symmetric form is presented below.

Corollary 8. The nominal system $E \dot{x}(t)=A x(t)$ is admissible if and only if there exist a positive definite matrix $P$ and $a$ matrix $Q$ with appropriate dimensions such that

$$
A\left(P E^{T}-S Q\right)+\left(P E^{T}-S Q\right)^{T} A^{T}<0
$$

where matrix $S \in R^{n \times n-m}$ is of full-column rank and satisfies $E S=0$.

Remark 9. The proposed admissibility condition in Lemma 7 or Corollary 8 for the nominal descriptor system is explicitly expressed by one compact form with a strict LMI and can be directly evaluated by the current LMI tool [40]. In contrast, some existing results need to embrace the additional nonstrict LMIs $E^{T} P=P^{T} E \geq 0$ [38] or $E^{T} Q E \geq 0$ [12] in their conditions which are intractable by the LMI solver and need extra treatments $[12,39]$.

The $D$-admissible issue of descriptor system $E \dot{x}(t)=$ $A x(t)$ is discussed in the sequel.

Lemma 10. The nominal descriptor system $E \dot{x}(t)=A x(t)$ is regular and impulse free, and all the poles lie in the region $H$ with $\alpha \geq 0$, shown in Figure 1, if and only if there exist a Hermitian matrix $P>0$ and a matrix $Q$ with appropriate dimensions such that

$$
\begin{aligned}
& {\left[e^{-j \theta}(A+\alpha E)\right]\left(P E^{T}-S Q\right)} \\
& \quad+\left(P E^{T}-S Q\right)^{*}\left[e^{-j \theta}(A+\alpha E)\right]^{*}<0,
\end{aligned}
$$

where matrix $S \in R^{n \times n-m}$ is of full-column rank and satisfies $E S=0$. 
Proof.

(Sufficiency). Suppose that there exist a Hermitian matrix $P>$ 0 and a matrix $Q$ satisfying (13). Deducing from the negative definite matrix of (13), it implies the nonsingularity property for $(A+\alpha E)$. Thus, we can find two nonsingular matrices $M_{1}$, $N_{1} \in R^{n \times n}$ such that

$$
\begin{gathered}
M_{1} E N_{1}=\left[\begin{array}{cc}
I_{m} & 0 \\
0 & 0
\end{array}\right], \\
M_{1}(A+\alpha E) N_{1}=\left[\begin{array}{cc}
A_{1}+\alpha I_{m} & A_{2} \\
A_{3} & A_{4}
\end{array}\right],
\end{gathered}
$$

where $A_{4}$ is nonsingular. From Definition 2 associated with Lemma 3, the system is ensured to be regular and impulse free. Thus, there exist two nonsingular matrices $M_{2}, N_{2} \in$ $R^{n \times n}[42]$ such that

$$
\begin{aligned}
M_{2} E N_{2} & =\left[\begin{array}{cc}
I_{m} & 0 \\
0 & 0
\end{array}\right], \\
M_{2}(A+\alpha E) N_{2} & =\left[\begin{array}{cc}
A_{1}^{\prime}+\alpha I_{m} & 0 \\
0 & I_{n-m}
\end{array}\right], \\
N_{2}^{-1} P N_{2}^{-T} & =\left[\begin{array}{cc}
P_{1} & P_{2} \\
P_{2}^{*} & P_{3}
\end{array}\right], \\
N_{2}^{-1} S Q M_{2}^{T} & =\left[\begin{array}{ll}
0 & 0 \\
S_{1} & S_{2}
\end{array}\right] .
\end{aligned}
$$

Substituting the above equations into (13) yields

$$
\begin{aligned}
& {\left[e^{-j \theta}(A+\alpha E)\right]\left(P E^{T}-S Q\right)+\left(P E^{T}-S Q\right)^{*}\left[e^{-j \theta}(A+\alpha E)\right]^{*}} \\
& \quad=M_{2}^{-1}\left\{e^{-j \theta}\left[\begin{array}{cc}
A_{1}^{\prime}+\alpha I_{m} & 0 \\
0 & I_{n-m}
\end{array}\right]\left[\begin{array}{cc}
P_{1} & 0 \\
P_{2}^{*}-S_{1} & -S_{2}
\end{array}\right]+\left[\begin{array}{cc}
P_{1} & P_{2}-S_{1}^{*} \\
0 & -S_{2}^{*}
\end{array}\right]\left[\begin{array}{cc}
A_{1}^{\prime}+\alpha I_{m} & 0 \\
0 & I_{n-m}
\end{array}\right] e^{j \theta}\right\} M_{2}^{-T} \\
& \quad=M_{2}^{-1}\left\{\left[\begin{array}{cc}
e^{-j \theta}\left(A_{1}^{\prime}+\alpha I_{m}\right) P_{1}+e^{j \theta} P_{1}\left(A_{1}^{\prime T}+\alpha I_{m}\right) & P_{2}-S_{1}^{*} \\
P_{2}^{*}-S_{1} & -e^{-j \theta} S_{2}-e^{j \theta} S_{2}^{*}
\end{array}\right]\right\} M_{2}^{-T}<0
\end{aligned}
$$

where (16) can imply

$$
\begin{aligned}
& e^{-j \theta}\left(A_{1}^{\prime}+\alpha I_{m}\right) P_{1}+e^{j \theta} P_{1}\left(A_{1}^{\prime T}+\alpha I_{m}\right) \\
& \quad=\left[e^{-j \theta}\left(A_{1}^{\prime}+\alpha I_{m}\right)\right] P_{1}+P_{1}\left[e^{-j \theta}\left(A_{1}^{\prime}+\alpha I_{m}\right)\right]^{*} \\
& \quad<0 .
\end{aligned}
$$

Since $P_{1}>0$, all the eigenvalues of $A_{1}^{\prime}$ are asserted to be located in the region $H$ according to Corollary 6; that is, all the poles of the nominal descriptor system locate in the region $H$.

(Necessity). Assume that the pair $(E, A)$ is regular and impulse free, and all its poles locate within the region $H$. There exist two nonsingular matrices $M_{3}, N_{3} \in R^{n \times n}$ such that

$$
M_{3} E N_{3}=\left[\begin{array}{cc}
I_{m} & 0 \\
0 & 0
\end{array}\right]
$$

$$
M_{3}(A+\alpha E) N_{3}=\left[\begin{array}{cc}
A_{1}^{\prime \prime}+\alpha I_{m} & 0 \\
0 & I_{n-m}
\end{array}\right] \text {. }
$$

Since all the eigenvalues of $A_{1}^{\prime \prime}$ lie in the region $H$, from Corollary 6 , there exists a Hermitian matrix $P_{1}^{\prime}>0$ such that

$$
\left[e^{-j \theta}\left(A_{1}^{\prime \prime}+\alpha I_{m}\right)\right] P_{1}^{\prime}+P_{1}^{\prime}\left[e^{-j \theta}\left(A_{1}^{\prime \prime}+\alpha I_{m}\right)\right]^{*}<0 .
$$

Let

$$
\begin{aligned}
P & =N_{3}^{-1}\left[\begin{array}{cc}
P_{1}^{\prime} & 0 \\
0 & P_{3}^{\prime}
\end{array}\right] N_{3}^{-T}>0, \\
N_{3}^{-1} S Q M_{3}^{T} & =\left[\begin{array}{ll}
0 & 0 \\
0 & S_{2}^{\prime}
\end{array}\right],
\end{aligned}
$$

with $P_{3}^{\prime}>0, e^{-j \theta} S_{2}^{\prime}+e^{j \theta} S_{2}^{\prime *}>0$. Substituting (18) and (20) into (13) we obtain

$$
\begin{aligned}
& {\left[e^{-j \theta}(A+\alpha E)\right]\left(P E^{T}-S Q\right)+\left(P E^{T}-S Q\right)^{*}\left[e^{-j \theta}(A+\alpha E)\right]^{*}} \\
& \quad=M_{3}^{-1}\left\{e^{-j \theta}\left[\begin{array}{cc}
A_{1}^{\prime \prime}+\alpha I_{m} & 0 \\
0 & I_{n-m}
\end{array}\right]\left[\begin{array}{cc}
P_{1}^{\prime} & 0 \\
0 & -S_{2}^{\prime}
\end{array}\right]+e^{j \theta}\left[\begin{array}{cc}
P_{1}^{\prime} & 0 \\
0 & -S_{2}^{\prime *}
\end{array}\right]\left[\begin{array}{cc}
A_{1}^{\prime \prime T}+\alpha I_{m} & 0 \\
0 & I_{n-m}
\end{array}\right]\right\} M_{3}^{-T}
\end{aligned}
$$




$$
=M_{3}^{-1}\left\{\left[\begin{array}{cc}
{\left[e^{-j \theta}\left(A_{1}^{\prime \prime}+\alpha I_{m}\right)\right] P_{1}^{\prime}+P_{1}^{\prime}\left[e^{-j \theta}\left(A_{1}^{\prime \prime}+\alpha I_{m}\right)\right]^{*}} & 0 \\
0 & -e^{-j \theta} S_{2}^{\prime}-e^{j \theta} S_{2}^{\prime *}
\end{array}\right]\right\} M_{3}^{-T}<0,
$$

and complete the proof.

The robust admissibility condition for the unforced descriptor system in (1), that is, $u(t) \equiv 0$ in (1), is addressed in the following.
Lemma 11. Assume $\widetilde{E}=E$ in (1). The unforced descriptor system in (1) with uncertainty Equation (3) is admissible, if and only if there exist a scalar $\alpha>0$ and matrices $P>0$ and $Q$ with appropriate dimensions such that

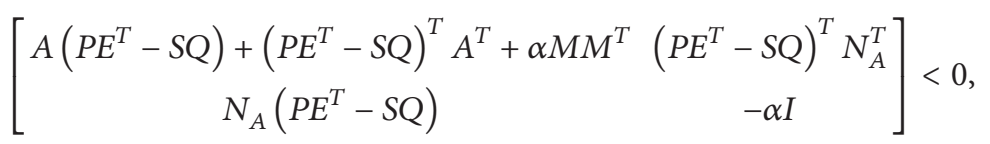

where matrix $S \in R^{n \times n-m}$ is of full-column rank and satisfies $E S=0$.

\section{Proof.}

(Sufficiency). Assume that there exist a scalar $\alpha>0$ and matrices $P>0$ and $Q$ satisfying (22). By the Schur complement [39], it is thus equivalent to

$$
\begin{aligned}
& A\left(P E^{T}-S Q\right)+\left(P E^{T}-S Q\right)^{T} A^{T}+\alpha M M^{T} \\
& \quad+\alpha^{-1}\left(P E^{T}-S Q\right)^{T} N_{A}^{T} N_{A}\left(P E^{T}-S Q\right)<0 .
\end{aligned}
$$

By Lemma 4, the above equation is equivalent to

$$
\begin{aligned}
A & \left(P E^{T}-S Q\right)+\left(P E^{T}-S Q\right)^{T} A^{T} \\
& +M D N_{A}\left(P E^{T}-S Q\right)+\left(P E^{T}-S Q\right)^{T}\left(M D N_{A}\right)^{T} \\
& =\left(A+M D N_{A}\right)\left(P E^{T}-S Q\right) \\
& +\left(P E^{T}-S Q\right)^{T}\left(A+M D N_{A}\right)^{T} \\
& =(A+\Delta A)\left(P E^{T}-S Q\right) \\
& +\left(P E^{T}-S Q\right)^{T}(A+\Delta A)^{T}<0 .
\end{aligned}
$$

The considered uncertain system is thus asserted to be admissible according to Corollary 8 .

(Necessity). Suppose that the unforced descriptor system (1) with $\widetilde{E}=E$ and uncertainties (3) is admissible. Based on Corollary 8 , there exist matrices $P>0$ and $Q$ such that

$$
\begin{aligned}
(A+ & \Delta A)\left(P E^{T}-S Q\right)+\left(P E^{T}-S Q\right)^{T}(A+\Delta A)^{T} \\
= & \left(A+M D N_{A}\right)\left(P E^{T}-S Q\right) \\
& +\left(P E^{T}-S Q\right)^{T}\left(A+M D N_{A}\right)^{T}<0 .
\end{aligned}
$$

By Lemma 4 , it is equivalent to

$$
\begin{aligned}
& A\left(P E^{T}-S Q\right)+\left(P E^{T}-S Q\right)^{T} A^{T}+\alpha M M^{T} \\
& \quad+\alpha^{-1}\left(P E^{T}-S Q\right)^{T} N_{A}^{T} N_{A}\left(P E^{T}-S Q\right)<0 .
\end{aligned}
$$

By the Schur complement, it thus is equivalent to

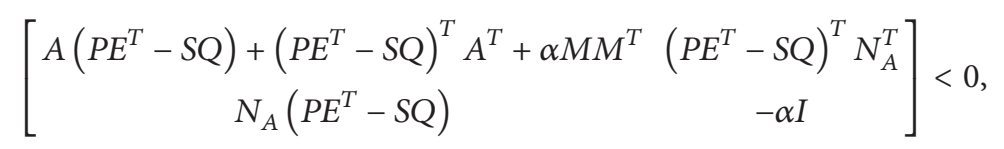

and the proof is completed.

Lemma 12. Assume $\widetilde{E}=E$ in (1). The unforced descriptor system in (1) with the uncertainty Equation (3) is regular, impulse free, and all the poles lie in the region $H$ with $\alpha \geq 0$, shown in Figure 1, if and only if there exist a scalar $\alpha>0$ and matrices $P>0$ and $Q$ with appropriate dimensions such that

$$
\left[\begin{array}{cc}
e^{-j \theta}(A+\alpha E)\left(P E^{T}-S Q\right)+\left(P E^{T}-S Q\right)^{*}\left[e^{-j \theta}(A+\alpha E)\right]^{*}+\alpha M M^{T} & \left(P E^{T}-S Q\right)^{*} N_{A}^{T} \\
N_{A}\left(P E^{T}-S Q\right) & -\alpha I
\end{array}\right]<0
$$


where matrix $S \in R^{n \times n-m}$ is of full-column rank and satisfies $E S=0$.

Proof.

(Sufficiency). Assume that there exist a scalar $\alpha>0$ and matrices $P>0$ and $Q$ satisfying (28). By the Schur complement, it is equivalent to

$$
\begin{aligned}
e^{-j \theta}(A & +\alpha E)\left(P E^{T}-S Q\right) \\
& +\left(P E^{T}-S Q\right)^{*}\left[e^{-j \theta}(A+\alpha E)\right]^{*}+\alpha M M^{T} \\
& +\alpha^{-1}\left(P E^{T}-S Q\right)^{*} N_{A}^{T} N_{A}\left(P E^{T}-S Q\right)<0 .
\end{aligned}
$$

By Lemma 4, the above equation is equivalent to

$$
\begin{aligned}
& e^{-j \theta}(A+\alpha E)\left(P E^{T}-S Q\right) \\
& +\left(P E^{T}-S Q\right)^{*}\left[e^{-j \theta}(A+\alpha E)\right]^{*} \\
& +e^{-j \theta}\left(M D N_{A}\right)\left(P E^{T}-S Q\right) \\
& +e^{j \theta}\left(P E^{T}-S Q\right)^{T}\left(M D N_{A}\right)^{T} \\
& =\left[e^{-j \theta}\left(A+M D N_{A}+\alpha E\right)\right]\left(P E^{T}-S Q\right) \\
& +\left(P E^{T}-S Q\right)^{*}\left[e^{-j \theta}\left(A+M D N_{A}+\alpha E\right)\right]^{*}
\end{aligned}
$$

$$
\begin{aligned}
& =\left[e^{-j \theta}(A+\Delta A+\alpha E)\right]\left(P E^{T}-S Q\right) \\
& +\left(P E^{T}-S Q\right)^{*}\left[e^{-j \theta}(A+\Delta A+\alpha E)\right]^{*}<0 .
\end{aligned}
$$

The considered uncertain system is thus asserted to be admissible according to Lemma 10.

(Necessity). Suppose that the unforced descriptor system (1) with $\widetilde{E}=E$ and uncertainties (3) is admissible. Based on Lemma 10 , there exist matrices $P>0$ and $Q$ such that

$$
\begin{aligned}
& {\left[e^{-j \theta}(A+\Delta A+\alpha E)\right]\left(P E^{T}-S Q\right)} \\
& \quad+\left(P E^{T}-S Q\right)^{*}\left[e^{-j \theta}(A+\Delta A+\alpha E)\right]^{*} \\
& \quad=\left[e^{-j \theta}\left(A+M D N_{A}+\alpha E\right)\right]\left(P E^{T}-S Q\right) \\
& \quad+\left(P E^{T}-S Q\right)^{*}\left[e^{-j \theta}\left(A+M D N_{A}+\alpha E\right)\right]^{*}<0 .
\end{aligned}
$$

By Lemma 4 for the above inequality, it is equivalent to

$$
\begin{aligned}
e^{-j \theta}(A & +\alpha E)\left(P E^{T}-S Q\right) \\
& +\left(P E^{T}-S Q\right)^{*}\left[e^{-j \theta}(A+\alpha E)\right]^{*}+\alpha M M^{T} \\
& +\alpha^{-1}\left(P E^{T}-S Q\right)^{*} N_{A}^{T} N_{A}\left(P E^{T}-S Q\right)<0 .
\end{aligned}
$$

By the Schur complement, we attain

$$
\left[\begin{array}{cc}
e^{-j \theta}(A+\alpha E)\left(P E^{T}-S Q\right)+\left(P E^{T}-S Q\right)^{*}\left[e^{-j \theta}(A+\alpha E)\right]^{*}+\alpha M M^{T} & \left(P E^{T}-S Q\right)^{*} N_{A}^{T} \\
N_{A}\left(P E^{T}-S Q\right) & -\alpha I
\end{array}\right]<0
$$

and complete the proof.

The admissibility and D-admissibility for the unforced descriptor system (1) with both uncertainties (2) and (3) are mainly proposed as follows.
Theorem 13. The unforced descriptor system (1) with uncertainties (2) and (3) is admissible, if there exist a scalar $\alpha>0$ and matrices $P>0$ and $Q$ with appropriate dimensions such that

$$
\left[\begin{array}{cc}
A\left(P E_{i}^{T}-S Q\right)+\left(P E_{i}^{T}-S Q\right)^{T} & A^{T}+\alpha M M^{T} \\
N_{A}\left(P E_{i}^{T}-S Q\right) & -\alpha I
\end{array}\right]<0 \quad \forall i
$$

where matrix $S \in R^{n \times n-m}$ is of full-column rank and satisfies $E_{i} S=0 \forall i$.

Proof. The proof can be derived from Lemma 10 associated with a set of vertices $E_{i}$ of the uncertain matrix $\widetilde{E}$ defined in (2).
Theorem 14. The unforced descriptor system in (1) with uncertainties (2) and (3) is regular and impulse free, and all the poles lie in the region $H$ with $\alpha \geq 0$, shown in Figure 1, if there exist a scalar $\alpha>0$ and matrices $P>0$ and $Q$ with appropriate dimensions such that 


$$
\left[\begin{array}{cc}
e^{-j \theta}\left(A+\alpha E_{i}\right)\left(P E_{i}^{T}-S Q\right)+\left(P E_{i}^{T}-S Q\right)^{*}\left[e^{-j \theta}\left(A+\alpha E_{i}\right)\right]^{*}+\alpha M M^{T} & \left(P E_{i}^{T}-S Q\right)^{*} N_{A}^{T} \\
N_{A}\left(P E_{i}^{T}-S Q\right) & -\alpha I
\end{array}\right]<0 \quad \forall i,
$$

where matrix $S \in R^{n \times n-m}$ is of full-column rank and satisfies $E_{i} S=0 \forall i$.

Proof. Assume that there exist a scalar $\alpha>0$ and matrices $P>0$ and $Q$ satisfying (35). Based on Lemma 12 associated with a set of vertices $E_{i}$ with the corresponding parameter $e_{i}$ defined in (2), one obtains

$$
\begin{aligned}
& {\left[\begin{array}{cc}
e^{-j \theta}(A+\alpha \widetilde{E})\left(P \widetilde{E}^{T}-S Q\right)+\left(P \widetilde{E}^{T}-S Q\right)^{*}\left[e^{-j \theta}(A+\alpha \widetilde{E})\right]^{*}+\alpha M M^{T} & \left(P \widetilde{E}^{T}-S Q\right)^{*} N_{A}^{T} \\
N_{A}\left(P \widetilde{E}^{T}-S Q\right) & -\alpha I
\end{array}\right]} \\
& =\left[\begin{array}{c}
e^{-j \theta}\left(A+\alpha \sum_{i} e_{i} E_{i}\right)\left(P\left(\sum_{i} e_{i} E_{i}\right)^{T}-S Q\right)+\left(P\left(\sum_{i} e_{i} E_{i}\right)^{T}-S Q\right)^{*}\left[e^{-j \theta}\left(A+\alpha \sum_{i} e_{i} E_{i}\right)\right]^{*}+\alpha M M^{T}\left(P\left(\sum_{i} e_{i} E_{i}\right)^{T}-S Q\right)^{*} N_{A}^{T} \\
N_{A}\left(P\left(\sum_{i} e_{i} E_{i}\right)^{T}-S Q\right)
\end{array}\right] \\
& =\sum_{i} e_{i}^{2}\left(\left[\begin{array}{cc}
e^{-j \theta}\left(A+\alpha E_{i}\right)\left(P E_{i}^{T}-S Q\right)+\left(P E_{i}^{T}-S Q\right)^{*}\left[e^{-j \theta}\left(A+\alpha E_{i}\right)\right]^{*}+\alpha M M^{T} & \left.\left(P E_{i}^{T}-S Q\right)^{*} N_{A}^{T}\right] \\
N_{A}\left(P E_{i}^{T}-S Q\right) & -\alpha I
\end{array}\right]\right)
\end{aligned}
$$

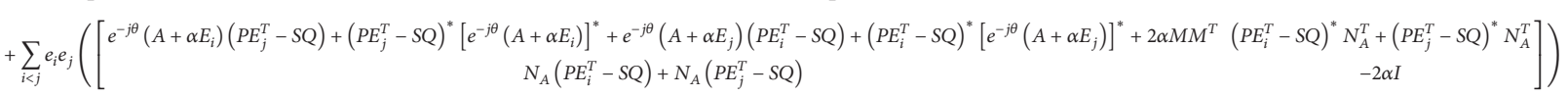

$$
\begin{aligned}
& \leq \sum_{i} e_{i}^{2}\left(\left[\begin{array}{cc}
e^{-j \theta}\left(A+\alpha E_{i}\right)\left(P E_{i}^{T}-S Q\right)+\left(P E_{i}^{T}-S Q\right)^{*}\left[e^{-j \theta}\left(A+\alpha E_{i}\right)\right]^{*}+\alpha M M^{T} & \left(P E_{i}^{T}-S Q\right)^{*} N_{A}^{T} \\
N_{A}\left(P E_{i}^{T}-S Q\right) & -\alpha I
\end{array}\right]\right) \\
& +\sum_{i<j} e_{i} e_{j}\left(\left[\begin{array}{cc}
e^{-j \theta}\left(A+\alpha E_{i}\right)\left(P E_{i}^{T}-S Q\right)+\left(P E_{i}^{T}-S Q\right)^{*}\left[e^{-j \theta}\left(A+\alpha E_{i}\right)\right]^{*}+e^{-j \theta}\left(A+\alpha E_{j}\right)\left(P E_{j}^{T}-S Q\right)+\left(P E_{j}^{T}-S Q\right)^{*}\left[e^{-j \theta}\left(A+\alpha E_{j}\right)\right]^{*}+2 \alpha M M^{T}\left(P E_{i}^{T}-S Q\right)^{*} N_{A}^{T}+\left(P E_{j}^{T}-S Q\right)^{*} N_{A}^{T} \\
N_{A}\left(P E_{i}^{T}-S Q\right)+N_{A}\left(P E_{j}^{T}-S Q\right) & -2 \alpha I
\end{array}\right]\right) .
\end{aligned}
$$

Deducing from (35), the above equation can be concluded to be negative definite. From Lemma 12, the unforced descriptor system in (1) with uncertainties (2) and (3) is asserted to be regular and impulse free, and all the poles lie in the region $H$.

Remark 15. Based on the result in Theorem 14, we can verify whether the uncertain descriptor system (1) is $D$-admissible via searching a set of feasible solutions $\alpha>0$ and matrices $P>$ 0 and $Q$ satisfying (35). Observing on (35), these equations provided for specific $D$-admissibility need to involve complex numbers, where the traditional LMI solver [40] cannot be applicable, and we can alternatively use a previous result [41], which suitably serves for solving these complex LMIs.

\section{Controller Design}

When the time-derivatives of the states are accessible in a control process, a PDSF control law can be represented as

$$
u(t)=K_{A} x(t)-K_{E} \dot{x}(t)=\widehat{K} z(t),
$$

where $\widehat{K}=\left[\begin{array}{ll}K_{A}-K_{E}\end{array}\right], z(t)=\left[\begin{array}{ll}x^{T}(t) & \dot{x}^{T}(t)\end{array}\right]^{T}$. According to the augmented state vector $z(t)$, system (1) can be transformed into

$$
\widehat{E} \dot{z}(t)=(\widetilde{\widehat{A}}+\Delta \widehat{A}) z(t)+(\widehat{B}+\Delta \widehat{B}) u(t),
$$

where

$$
\begin{aligned}
\widehat{E} & =\left[\begin{array}{ll}
I_{n} & 0 \\
0 & 0
\end{array}\right], \\
\widetilde{A} & =\left[\begin{array}{cc}
0 & I_{n} \\
A & -\widetilde{E}
\end{array}\right], \\
\widehat{B} & =\left[\begin{array}{l}
0 \\
B
\end{array}\right], \\
\widehat{M} & =\left[\begin{array}{c}
0 \\
M
\end{array}\right], \\
\widehat{N}_{A} & =\left[\begin{array}{ll}
N_{A} & 0
\end{array}\right], \\
\widehat{N}_{B} & =N_{B} .
\end{aligned}
$$

By the augmented descriptor system (38) equipped with the PDSF controller (37), we present the design condition for the resulting closed-loop system as follows.

Theorem 16. For system (38) associated with the PDSF control law in (37), the resulting closed-loop uncertain descriptor system is admissible, if there exist a scalar $\alpha>0$ and, matrices $P>0, Q, F_{A}$, and $F_{E}$ with appropriate dimensions such that 


$$
\left[\begin{array}{ccc}
Q+Q^{T} & P A^{T}+F_{A}^{T} B^{T}-Q^{T} E_{i}^{T}-F_{E}^{T} B^{T}-Q & P N_{A}^{T}+F_{A}^{T} N_{B}^{T}-F_{E}^{T} N_{B}^{T} \\
A P+B F_{A}-E_{i} Q-B F_{E}-Q^{T} & E_{i} Q+Q^{T} E_{i}^{T}+B F_{E}+F_{E}^{T} B^{T}+\alpha M M^{T} & F_{E}^{T} N_{B}^{T} \\
N_{A} P+N_{B} F_{A}-N_{B} F_{E} & N_{B} F_{E} & -\alpha I
\end{array}\right]<0 \quad \forall i .
$$

Then, a set of normalizing and stabilizing PDSF gains in (37) is given by $\widehat{K}=\left[\begin{array}{ll}K_{A} & -K_{E}\end{array}\right]=\left[\begin{array}{ll}F_{A} P^{-1} & -F_{E} Q^{-1}\end{array}\right]$.

Proof. Substituting the PDSF controller (37) into the augmented system (38), a resulting closed-loop system can be described as

$$
\begin{aligned}
& {\left[\begin{array}{cc}
I_{n} & 0 \\
0 & 0
\end{array}\right] \dot{z}(t)} \\
& \quad=\left(\widetilde{\widetilde{A}}+\widehat{M} \widehat{D} \widehat{N}_{A}+\widehat{B} \widehat{K}+\widehat{M} \widehat{D} \widehat{N}_{B} \widehat{K}\right) z(t) .
\end{aligned}
$$

According to the compatible form of the block-matrix form in (39), we let

$$
\begin{aligned}
& \widehat{P}=\left[\begin{array}{ll}
P & Q^{T} \\
Q & P
\end{array}\right]>0, \\
& \widehat{S}=\left[\begin{array}{l}
0 \\
I_{n}
\end{array}\right], \\
& \widehat{Q}=\left[\begin{array}{ll}
0 & Q
\end{array}\right]
\end{aligned}
$$

with $P>0$ and $P>0$ satisfying $P-Q^{T} P Q>0$ and

$$
\widehat{P} \widehat{E}^{T}-\widehat{S} \widehat{Q}=\left[\begin{array}{cc}
P & 0 \\
Q & -Q
\end{array}\right] .
$$

According to Corollary 8 for the augmented system (41), by (12) we can verify the admissibility of system (41) by

$$
\begin{aligned}
& \left(\widetilde{\widetilde{A}}+\widehat{B} \widehat{K}+\widehat{M} \widehat{D} \widehat{N}_{A}+\widehat{M} \widehat{D} \widehat{N}_{B} \widehat{K}\right)\left(\widehat{P} \widehat{E}^{T}-\widehat{S} \widehat{Q}\right) \\
& +\left(\widehat{P} \widehat{E}^{T}-\widehat{S} \widehat{Q}\right)^{T} \\
& \cdot\left(\widetilde{\widetilde{A}}+\widehat{B} \widehat{K}+\widehat{M} \widehat{D} \widehat{N}_{A}+\widehat{M} \widehat{D} \widehat{N}_{B} \widehat{K}\right)^{T}=(\widetilde{\widetilde{A}}+\widehat{B} \widehat{K}) \\
& \cdot\left(\widehat{P} \widehat{E}^{T}-\widehat{S} \widehat{Q}\right)+\left(\widehat{P} \widehat{E}^{T}-\widehat{S} \widehat{Q}\right)^{T}(\widetilde{\widetilde{A}}+\widehat{B} \widehat{K})^{T} \\
& +\widehat{M} \widehat{D}\left(\widehat{N}_{A}+\widehat{N}_{B} \widehat{K}\right)\left(\widehat{P} \widehat{E}^{T}-\widehat{S} \widehat{Q}\right)+\left(\widehat{P} \widehat{E}^{T}-\widehat{S} \widehat{Q}\right)^{T} \\
& \cdot\left(\widehat{N}_{A}+\widehat{N}_{B} \widehat{K}\right)^{T} \widehat{D}^{T} \widehat{M}^{T}<0 .
\end{aligned}
$$

And, by Lemma 4, it is equivalent to

$$
\begin{gathered}
(\widetilde{\widetilde{A}}+\widehat{B} \widehat{K})\left(\widehat{P} \widehat{E}^{T}-\widehat{S} \widehat{Q}\right)+\left(\widehat{P} \widehat{E}^{T}-\widehat{S} \widehat{Q}\right)^{T}(\widetilde{\widetilde{A}}+\widehat{B} \widehat{K})^{T} \\
+\alpha \widehat{M} \widehat{M}^{T}+\alpha^{-1}\left(\widehat{P} \widehat{E}^{T}-\widehat{S} \widehat{Q}\right)^{T}\left(\widehat{N}_{A}+\widehat{N}_{B} \widehat{K}\right)^{T} \\
\cdot\left(\widehat{N}_{A}+\widehat{N}_{B} \widehat{K}\right)\left(\widehat{P} \widehat{E}^{T}-\widehat{S} \widehat{Q}\right)<0
\end{gathered}
$$

and using the Schur complement, it leads to

$$
\left[\begin{array}{cc}
(\widetilde{\widehat{A}}+\widehat{B} \widehat{K})\left(\widehat{P} \widehat{E}^{T}-\widehat{S} \widehat{Q}\right)+\left(\widehat{P} \widehat{E}^{T}-\widehat{S} \widehat{Q}\right)^{T}(\widetilde{\widehat{A}}+\widehat{B} \widehat{K})^{T}+\alpha \widehat{M}^{T} \widehat{M}^{T}\left(\widehat{P} \widehat{E}^{T}-\widehat{S} \widehat{Q}\right)^{T}\left(\widehat{N}_{A}+\widehat{N} \widehat{N}_{B} \widehat{K}\right)^{T} \\
\left(\widehat{N}_{A}+\widehat{N}_{B} \widehat{K}\right)\left(\widehat{P} \widehat{E}^{T}-\widehat{S} \widehat{Q}\right) & -\alpha I
\end{array}\right]<0
$$

Thus, according to Corollary 8 , if there exist a scalar $\alpha>0$ and matrices $\widehat{P}, \widehat{S}, \widehat{Q}$ in (42), and $\widehat{K}$ in (37) satisfying (46), system (41) with the PDSF controller is admissible. In the sequel, we will deduce that once (40) is satisfied, it can consequently imply (46).

Let $\widehat{K}=\left[\begin{array}{ll}K_{A} & -K_{E}\end{array}\right]=\left[\begin{array}{ll}F_{A} P^{-1} & -F_{E} Q^{-1}\end{array}\right]$. Substituting (37), (39), and (43) into the block $(1,1)$ of $(46)$ leads to

$$
\begin{aligned}
& (\widetilde{\widetilde{A}}+\widehat{B} \widehat{K})\left(\widehat{P} \widehat{E}^{T}-\widehat{S} \widehat{Q}\right)+\left(\widehat{P} \widehat{E}^{T}-\widehat{S} \widehat{Q}\right)^{T}(\widetilde{\widetilde{A}}+\widehat{B} \widehat{K})^{T}+\alpha \widehat{M} \widehat{M}^{T}=\left[\begin{array}{cc}
0 & I \\
A+B K_{A} & -\widetilde{E}-B K_{E}
\end{array}\right]\left[\begin{array}{cc}
P & 0 \\
Q & -Q
\end{array}\right] \\
& +\left[\begin{array}{cc}
P & Q^{T} \\
0 & -Q^{T}
\end{array}\right]\left[\begin{array}{cc}
0 & A^{T}+K_{A}^{T} B^{T} \\
I & -\widetilde{E}^{T}-K_{E}^{T} B^{T}
\end{array}\right]+\alpha\left[\begin{array}{c}
0 \\
M
\end{array}\right]\left[\begin{array}{ll}
0 & M^{T}
\end{array}\right]
\end{aligned}
$$




$$
\begin{aligned}
& =\left[\begin{array}{cc}
Q+Q^{T} & P A^{T}+P K_{A}^{T} B^{T}-Q^{T} \widetilde{E}^{T}-Q^{T} K_{E}^{T} B^{T}-Q \\
A P+B K_{A} P-\widetilde{E} Q-B K_{E} Q-Q^{T} & \widetilde{E} Q+Q^{T} \widetilde{E}^{T}+B K_{E} Q+Q^{T} K_{E}^{T} B^{T}+\alpha M M^{T}
\end{array}\right] \\
& =\left[\begin{array}{cc}
Q+Q^{T} & P A^{T}+F_{A}^{T} B^{T}-Q^{T} \widetilde{E}^{T}-F_{E}^{T} B^{T}-Q \\
A P+B F_{A}-\widetilde{E} Q-B F_{E}-Q^{T} & \widetilde{E} Q+Q^{T} \widetilde{E}^{T}+B F_{E}+F_{E}^{T} B^{T}+\alpha M M^{T}
\end{array}\right] .
\end{aligned}
$$

Similarly, substituting (37), (39), and (43) into the block $(2,1)$ of (46) yields

$$
\begin{aligned}
& \left(\widehat{N}_{A}+\widehat{N}_{B} \widehat{K}\right)\left(\widehat{P} \widehat{E}^{T}-\widehat{S} \widehat{Q}\right) \\
& =\left(\left[\begin{array}{ll}
N_{A} & 0
\end{array}\right]+\left[\begin{array}{ll}
N_{B} K_{A} & -N_{B} K_{E}
\end{array}\right]\right)\left[\begin{array}{cc}
P & 0 \\
Q & -Q
\end{array}\right] \\
& =\left[\begin{array}{ll}
N_{A} P+N_{B} K_{A} P-N_{B} K_{E} Q & N_{B} K_{E} Q
\end{array}\right] \\
& =\left[\begin{array}{ll}
N_{A} P+N_{B} F_{A}-N_{B} F_{E} & N_{B} F_{E}
\end{array}\right] .
\end{aligned}
$$

When multiplying $e_{i}$ to the individual inequality in (40) with respect to $E_{i}$ and then summing all of them, the integrated one equation can attain $(46)$ with the inner blocks $(1,1)$ and $(2,1)$ that are replaced by $(47)$ and (48), respectively. Thus, the closed-loop descriptor system (41) with the PDSF controller (37) is admissible for all the allowable uncertainties (2) and (3).

\section{Illustrative Examples}

In this section, we present two numerical examples to demonstrate the superiority and effectiveness of the proposed approach.

Example 1 (see [12]). Consider a simple circuit network as drawn in Figure 2, where $V_{S}(t)$ is a voltage source, $R, L$, and $C$ represent the resistor, inductor, and capacity, respectively, and their voltages are correspondingly denoted by $V_{R}(t), V_{L}(t)$, and $V_{C}(t)$. By Kirchoff's laws, a circuit model can be formed as

$$
\begin{gathered}
{\left[\begin{array}{llll}
L & 0 & 0 & 0 \\
0 & 0 & 1 & 0 \\
0 & 0 & 0 & 0 \\
0 & 0 & 0 & 0
\end{array}\right]\left[\begin{array}{c}
\dot{I}^{(}(t) \\
\dot{V}_{L}(t) \\
\dot{V}_{C}(t) \\
\dot{V}_{R}(t)
\end{array}\right]} \\
=\left[\begin{array}{cccc}
0 & 1 & 0 & 0 \\
\frac{1}{C} & 0 & 0 & 0 \\
-R & 0 & 0 & 1 \\
0 & 1 & 1 & 1
\end{array}\right]\left[\begin{array}{c}
I(t) \\
V_{L}(t) \\
V_{C}(t) \\
V_{R}(t)
\end{array}\right]+\left[\begin{array}{c}
0 \\
0 \\
0 \\
-1
\end{array}\right] V_{S}(t) .
\end{gathered}
$$

In practical design and application, the inaccuracy rates of the circuit components need to be considered. Thus, the uncertain components' values in (49) are denoted as $R=$ $1 \pm 20 \%, L=1 \pm 10 \%$, and $C=1 \pm 10 \%$.

For the unforced system (49), that is, $V_{S}(t) \equiv 0$ in (49), with the given uncertain parameters, the regarded unforced system is specified to verify whether it is $D$-admissible with the poles within the desired region shown in Figure 3, that is, the intersection of three open-half planes $H_{1}, H_{2}$, and $H_{3}$, determined by $L_{1}\left(a_{1}=-0.3, \theta_{1}=0^{\circ}\right), L_{2}\left(a_{2}=0, \theta_{2}=\right.$ $\left.20^{\circ}\right)$, and $L_{3}\left(a_{3}=0, \theta_{3}=-20^{\circ}\right)$, respectively. Since the derivative matrix in (49) is insufficient rank and embraces the parametric uncertainty, the existing results [29-31, 36] are not applicable. For the considered system, we also cannot conclude the poles' location within the prescribed region by the previous approach [12], where when we performed the evaluating algorithm by [12], it cannot attain a convergent solution.

However, based on the proposed approach in Theorem 14 for the uncertain system (49), two vertices of the perturbed derivative matrix can be denoted as

$$
\begin{aligned}
& E_{1}=\left[\begin{array}{llll}
0.9 & 0 & 0 & 0 \\
0 & 0 & 1 & 0 \\
0 & 0 & 0 & 0 \\
0 & 0 & 0 & 0
\end{array}\right], \\
& E_{2}=\left[\begin{array}{llll}
1.1 & 0 & 0 & 0 \\
0 & 0 & 1 & 0 \\
0 & 0 & 0 & 0 \\
0 & 0 & 0 & 0
\end{array}\right],
\end{aligned}
$$

and the uncertain system matrix is represented by normbounded uncertainties with

$$
\begin{aligned}
A & =\left[\begin{array}{cccc}
0 & 1 & 0 & 0 \\
\frac{100}{99} & 0 & 0 & 0 \\
-1 & 0 & 0 & 1 \\
0 & 1 & 1 & 1
\end{array}\right], \\
M & =\left[\begin{array}{c}
0 \\
\frac{100}{99} \\
1 \\
0
\end{array}\right], \\
N_{A} & =\left[\begin{array}{llll}
0.1 & 0 & 0 & 0
\end{array}\right] .
\end{aligned}
$$


Thus, by initially giving a matrix $S=\left[\begin{array}{llll}0 & 1 & 0 & 0 \\ 0 & 0 & 0 & 1\end{array}\right]^{T}$ satisfying $E_{1} S=E_{2} S=0$, an admissibility criterion can be constructed by a set of LMIs from (35). When evaluating them via the previous result [41], a set of feasible solutions is obtained as

$$
\begin{aligned}
\alpha & =2.3367 \times 10^{-2}>0 \\
P & =\left[\begin{array}{cccc}
58.8167 & -12.8561+0.0044 j & -24.3097+0.0038 j & 13.3715-0.0007 j \\
-12.8561-0.0044 j & 100 & -13.6350-0.0029 j & 0 \\
-24.3097-0.0038 j & -13.6350+0.0029 j & 66.8015 & -7.3815-0.0003 j \\
13.3715+0.0007 j & 0 & -7.3815+0.0003 j & 100
\end{array}\right] \times 10^{-2}>0, \\
Q & =\left[\begin{array}{cccc}
-26.0836-0.0088 j & -27.2699-0.0058 j & 13.4203+0.0242 j & -4.7326-0.0010 j \\
27.1906+0.0024 j & -14.7627+0.0005 j & -12.5834-0.0180 j & -18.8938+0.0024 j
\end{array}\right] \times 10^{-2} .
\end{aligned}
$$

Therefore, the considered electrical circuit system (49) is asserted to be $D$-admissible according to Theorem 14 .

In the next example, the PDSF control law (37) is involved for stabilizing an uncertain closed-loop descriptor system.

Example 2. Suppose an uncertain descriptor system as (1) with uncertainties (2) and (3) given as

$$
\begin{aligned}
& \widetilde{E}=\left[\begin{array}{cccc}
2+\omega & 0 & 1 \\
1 & 1 & 0 \\
0 & 0 & 0
\end{array}\right], \\
& A=\left[\begin{array}{lll}
0 & 0 & 2 \\
0 & 0 & 1 \\
0 & 1 & 5
\end{array}\right], \\
& B=\left[\begin{array}{l}
0 \\
1 \\
1
\end{array}\right], \\
& M=\left[\begin{array}{l}
0.6 \\
0.5 \\
0.5
\end{array}\right], \\
& N_{A}=\left[\begin{array}{lll}
0.7 & 0.5 & 0.6
\end{array}\right] \text {, } \\
& N_{B}=0.5 \text {, }
\end{aligned}
$$

where the uncertain parameter is bounded as $|\omega| \leq 1.5$.

In this system, the $\sigma(E, A)$ of the nominal system do not lie in the open left half plane: that is, the unforced system is not admissible, and a stabilizing control law needs to be implemented. But, because the considered system includes the perturbed derivative matrix $\widetilde{E}$, the previous results $[9,17$, 30, 31] are not applicable. Furthermore, when we evaluated the constructed LMI conditions by the previous approach [36], the evaluation process of the LMI solver is eventually infeasible.
However, based on the proposed approach in Theorem 16, two vertices of the perturbed matrix $\widetilde{E}$ can be denoted as

$$
\begin{aligned}
& E_{1}=\left[\begin{array}{lll}
0.5 & 0 & 1 \\
1 & 1 & 0 \\
0 & 0 & 0
\end{array}\right], \\
& E_{2}=\left[\begin{array}{lll}
3.5 & 0 & 1 \\
1 & 1 & 0 \\
0 & 0 & 0
\end{array}\right] .
\end{aligned}
$$

By involving the PDSF controller (37), we correspondingly construct a set of strict LMIs by (40). Numerically evaluating them via the LMI solver, we then obtain a set of feasible solutions as

$$
\begin{aligned}
\alpha & =1745.6663 \\
P & =\left[\begin{array}{rrr}
2.9101 & 3.8259 & -3.2174 \\
3.8259 & 141.4395 & -3.5346 \\
-3.3274 & -3.5346 & 3.5953
\end{array}\right] \times 10^{2}>0, \\
Q & =\left[\begin{array}{ccc}
-1.2323 & -8.6101 & 9.0213 \\
3.6838 & -73.7325 & 10.9194 \\
-4.2563 & 14.1596 & -15.5481
\end{array}\right] \times 10^{2}, \\
F_{A} & =\left[\begin{array}{lll}
0.6904 & -105.2891 & -29.6484
\end{array}\right] \times 10^{2}, \\
F_{E} & =\left[\begin{array}{lll}
-4.3797 & 30.1063 & -30.1599
\end{array}\right] \times 10^{2} .
\end{aligned}
$$

And, a set of stabilizing PDSF gains is determined by

$$
\begin{aligned}
& K_{A}=F_{A} P^{-1}=\left[\begin{array}{lll}
-910.0170 & 3.3977 & -819.2624
\end{array}\right], \\
& K_{E}=F_{E} Q^{-1}=\left[\begin{array}{lll}
-1.0537 & -0.0349 & 1.3039
\end{array}\right] .
\end{aligned}
$$

Thus, the resulting closed-loop descriptor system equipped with the achieved PDSF controller $u(t)=K_{A} x(t)-K_{E} \dot{x}(t)$ is asserted to be robustly admissible according to Theorem 16 . 


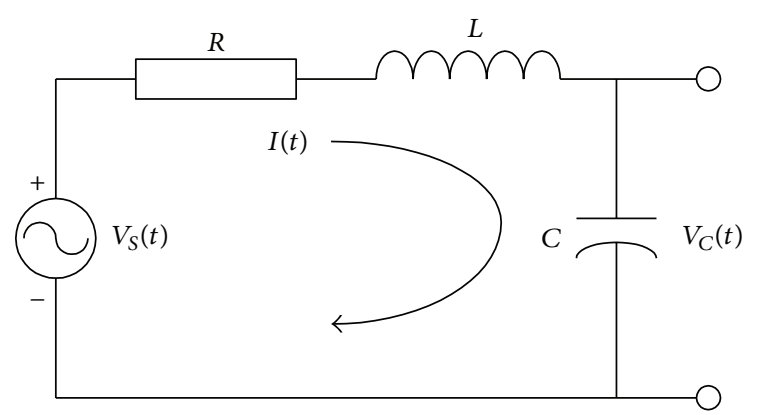

Figure 2: A circuit network for Example 1.

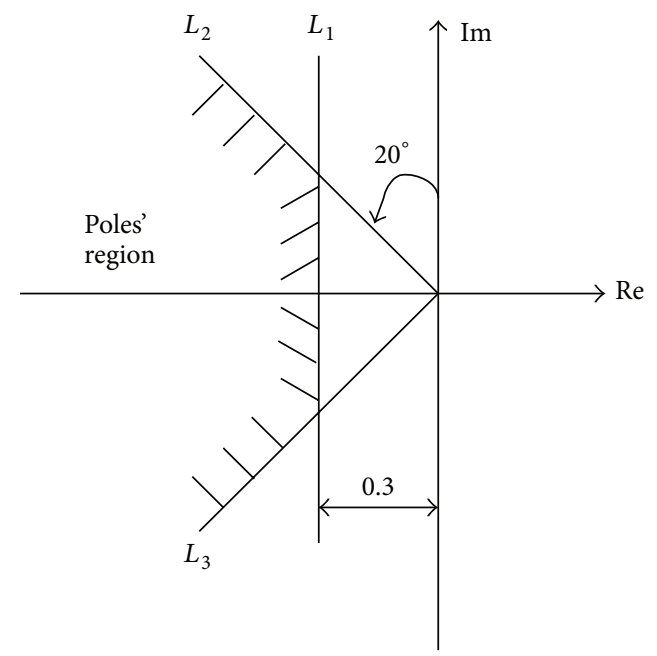

FIgURE 3: The prescribed pole region for Example 1.

\section{Conclusions}

The $D$-admissibility analysis and the controller design for descriptor systems with parametric uncertainty in the derivative matrix have been studied in this work. Based on the matrix theory, the equivalent admissibility and $D$ admissibility criteria that could be expressed by compact and refined LMI form for the nominal descriptor system were originally derived. Then, the admissibility and $D$ admissibility criteria for the unforced system with the uncertainties simultaneously existing in the derivative matrix and the system matrices thus could be attained. It is worthy to mention that the proposed distinct forms no longer have to involve additional nonstrict LMIs $E^{T} P=P E^{T} \geq 0$ or $E^{T} Q E \geq 0$ for the descriptor systems, which are intractable by existing LMI solvers. Furthermore, when involving the PDSF control law, we further discussed the robust controller design for the resulting closed-loop descriptor systems. Since all the proposed criteria could be formulated by LMIs, we thus applied the existing LMI solvers for numerical evaluation. Finally, the illustrated examples demonstrated that the proposed methods are valid and practicable.

\section{Conflict of Interests}

The authors declare that there is no conflict of interests regarding the publication of this paper.

\section{Acknowledgment}

The work was partially supported by the Ministry of Science and Technology of the Republic of China under the Contract MOST 104-2221-E-845-004.

\section{References}

[1] D. G. Luenberger, "Dynamic equations in descriptor form," IEEE Transactions on Automatic Control, vol. 22, no. 3, pp. 312321, 1977.

[2] F. L. Lewis, "A survey of linear singular systems," Circuits, Systems, and Signal Processing, vol. 5, no. 1, pp. 3-36, 1986.

[3] L. Jódar and P. Merello, "Positive solutions of discrete dynamic Leontief input-output model with possibly singular capital matrix," Mathematical and Computer Modelling, vol. 52, no. 78, pp. 1081-1087, 2010.

[4] Y.-Y. Cao and Z. Lin, "A descriptor system approach to robust stability analysis and controller synthesis," IEEE Transactions on Automatic Control, vol. 49, no. 11, pp. 2081-2084, 2004.

[5] J.-H. Chou and W.-H. Liao, "Stability robustness of continuoustime perturbed descriptor systems," IEEE Transactions on Circuits and Systems I: Fundamental Theory and Applications, vol. 46, no. 9, pp. 1153-1155, 1999.

[6] G. Zong, L. Hou, and J. Li, "A descriptor system approach to $l_{2}-l_{\infty}$ filtering for uncertain discrete-time switched system with mode-dependent time-varying delays," International Journal of Innovative Computing, Information and Control A, vol. 7, no. 5, pp. 2213-2224, 2011.

[7] D. L. Chu and D. W. C. Ho, "Necessary and sufficient conditions for the output feedback regularization of descriptor systems," IEEE Transactions on Automatic Control, vol. 44, no. 2, pp. 405412, 1999.

[8] S. Xu and C. Yang, "Robust stabilization for generalized statespace systems with uncertainty," International Journal of Control, vol. 72, no. 18, pp. 1659-1664, 1999.

[9] S. Xu, P. Van Dooren, R. Stefan, and J. Lam, "Robust stability and stabilization for singular systems with state delay and parameter uncertainty," IEEE Transactions on Automatic Control, vol. 47, no. 7, pp. 1122-1128, 2002.

[10] C. Yang, Q. Zhang, Y. Lin, and L. Zhou, "Positive realness and absolute stability problem of descriptor systems," IEEE Transactions on Circuits and Systems. I. Regular Papers, vol. 54, no. 5, pp. 1142-1149, 2007.

[11] M. S. Mahmoud, "Delay-dependent dissipativity of singular time-delay systems," IMA Journal of Mathematical Control and Information, vol. 26, no. 1, pp. 45-58, 2009.

[12] C.-P. Huang, "Pole location clustering for uncertain descriptor systems with perturbed derivative matrices," International Journal of Innovative Computing, Information and Control, vol. 7, no. 7, pp. 3597-3613, 2011.

[13] X.-Z. Dong, "Admissibility analysis of linear singular systems via a delta operator method," International Journal of Systems Science, vol. 45, no. 11, pp. 2366-2375, 2014. 
[14] Y.-J. Liu and S. Tong, "Adaptive fuzzy control for a class of nonlinear discrete-time systems with backlash," IEEE Transactions on Fuzzy Systems, vol. 22, no. 5, pp. 1359-1365, 2014.

[15] Y.-J. Liu and S. Tong, "Adaptive NN tracking control of uncertain nonlinear discrete-time systems with nonaffine dead-zone input," IEEE Transactions on Cybernetics, vol. 45, no. 3, pp. 497505, 2015.

[16] Y.-J. Liu and S. Tong, "Adaptive fuzzy identification and control for a class of nonlinear pure-feedback MIMO systems with unknown dead zones,' IEEE Transactions on Fuzzy Systems, vol. 23, no. 5, pp. 1387-1398, 2015.

[17] M. Chaabane, O. Bachelier, M. Souissi, and D. Mehdi, "Stability and stabilization of continuous descriptor systems: an LMI approach," Mathematical Problems in Engineering, vol. 2006, Article ID 39367, 15 pages, 2006.

[18] F. A. Faria, E. Assunção, M. C. Teixeira, and R. Cardim, "Robust state-derivative feedback LMI-based designs for linear descriptor systems," Mathematical Problems in Engineering, vol. 2010, Article ID 927362, 15 pages, 2010.

[19] Y. Chen, T. Xu, C. Zeng, Z. Zhou, and Q. Zhang, "Simultaneous stabilization for uncertain descriptor systems with input saturation," International Journal of Robust and Nonlinear Control, vol. 22, no. 17, pp. 1938-1951, 2012.

[20] C. Lin, J. L. Wang, G.-H. Yang, and J. Lam, "Robust stabilization via state feedback for descriptor systems with uncertainties in the derivative matrix," International Journal of Control, vol. 73, no. 5, pp. 407-415, 2000.

[21] C. Lin, J. Lam, J. Wang, and G.-H. Yang, "Analysis on robust stability for interval descriptor systems," Systems and Control Letters, vol. 42, no. 4, pp. 267-278, 2001.

[22] K. Furuta and S. B. Kim, "Pole assignment in a specified disk," IEEE Transactions on Automatic Control, vol. 32, no. 5, pp. 423427, 1987.

[23] M. Chilali and P. Gahinet, " $H_{\infty}$ design with pole placement constraints: an LMI approach," IEEE Transactions on Automatic Control, vol. 41, no. 3, pp. 358-367, 1996.

[24] Y.-T. Juang, Z.-C. Hong, and Y. T. Wang, "Robustness of pole-assignment in a specified region," IEEE Transactions on Automatic Control, vol. 34, no. 7, pp. 758-760, 1989.

[25] M. Chilali, P. Gahinet, and P. Apkarian, "Robust pole placement in LMI regions," IEEE Transactions on Automatic Control, vol. 44, no. 12, pp. 2257-2270, 1999.

[26] D. Peaucelle, D. Arzelier, O. Bachelier, and J. Bernussou, "A new robust $\mathscr{D}$-stability condition for real convex polytopic uncertainty," Systems \& Control Letters, vol. 40, no. 1, pp. 21-30, 2000.

[27] M. Saeki, "On the pole location of a system designed by robust stability-degree assignment," Systems and Control Letters, vol. 42 , no. 5, pp. 353-361, 2001.

[28] X. Liu, "A new method for the pole estimation of linear timeinvariant systems using singular value decomposition," Journal of Sound and Vibration, vol. 310, no. 4-5, pp. 998-1013, 2008.

[29] Y. Bavafa-Toosi, H. Ohmori, and B. Labibi, "Note on finite eigenvalues of regular descriptor systems," IEE ProceedingsControl Theory \& Applications, vol. 153, no. 4, pp. 502-503, 2006.

[30] O. Rejichi, O. Bachelier, M. Chaabane, and D. Mehdi, "Robust root-clustering analysis in a union of subregions for descriptor systems," IET Control Theory \& Applications, vol. 2, no. 7, pp. 615-624, 2008.

[31] B. Zhang, "Eigenvalue assignment in linear descriptor systems via output feedback," IET Control Theory and Applications, vol. 7, no. 15, pp. 1906-1913, 2013.
[32] R. Mukundan and W. Dayawansa, "Feedback control of singular systems-proportional and derivative feedback of the state," International Journal of Systems Science, vol. 14, no. 6, pp. 615632, 1983.

[33] A. Bunse-Gerstner, V. Mehrmann, and N. K. Nichols, "Regularization of descriptor systems by derivative and proportional state feedback," SIAM Journal on Matrix Analysis and Applications, vol. 13, no. 1, pp. 46-67, 1992.

[34] V. X. Le, "Synthesis of proportional-plus-derivative feedbacks for descriptor systems," IEEE Transactions on Automatic Control, vol. 37, no. 5, pp. 672-675, 1992.

[35] G.-R. Duan and R. J. Patton, "Robust pole assignment in descriptor systems via proportional plus partial derivative state feedback," International Journal of Control, vol. 72, no. 13, pp. 1193-1203, 1999.

[36] C. Lin, Q.-G. Wang, and T. H. Lee, "Robust normalization and stabilization of uncertain descriptor systems with normbounded perturbations," IEEE Transactions on Automatic Control, vol. 50, no. 4, pp. 515-520, 2005.

[37] G.-R. Duan and H.-H. Yu, "Robust pole assignment in highorder descriptor linear systems via proportional plus derivative state feedback," IET Control Theory \& Applications, vol. 2, no. 4, pp. 277-287, 2008.

[38] I. Masubuchi, Y. Kamitane, A. Ohara, and N. Suda, " $H_{\infty}$ control for descriptor systems: a matrix inequalities approach," Automatica, vol. 33, no. 4, pp. 669-673, 1997.

[39] S. Boyd, L. E. Ghaoui, E. Feron, and V. Balakrishnan, Linear Matrix Inequalities in System and Control Theory, SIAM, Philadelphia, Pa, USA, 1994.

[40] P. Gahinet, A. Nemirovski, A. J. Jaub, and M. Chilali, LMI Control Toolbox User's Guide, The Mathworks Partner Series, The Math Works Inc, Natick, Mass, USA, 1995.

[41] C.-P. Huang, Y.-T. Juang, and H.-L. Lin, "A complex projection scheme and applications," Computers and Mathematics with Applications, vol. 49, no. 4, pp. 515-524, 2005.

[42] L. Dai, Singular Control Systems, vol. 118 of Lecture Notes in Control and Information Sciences, Springer, New York, NY, USA, 1989.

[43] I. R. Petersen, "A stabilization algorithm for a class of uncertain linear systems," Systems \& Control Letters, vol. 8, no. 4, pp. 351357, 1987.

[44] C.-P. Huang, "Stability analysis and controller synthesis for fuzzy descriptor systems," International Journal of Systems Science, vol. 44, no. 1, pp. 23-33, 2013. 


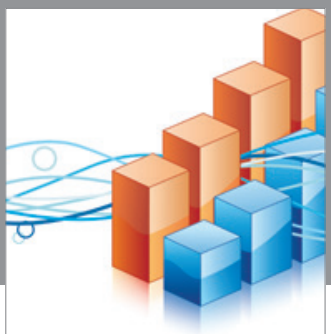

Advances in

Operations Research

vatem alat4

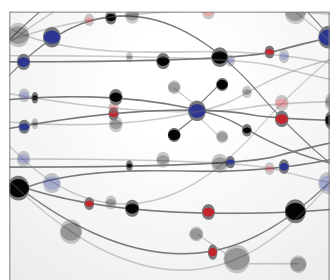

\section{The Scientific} World Journal
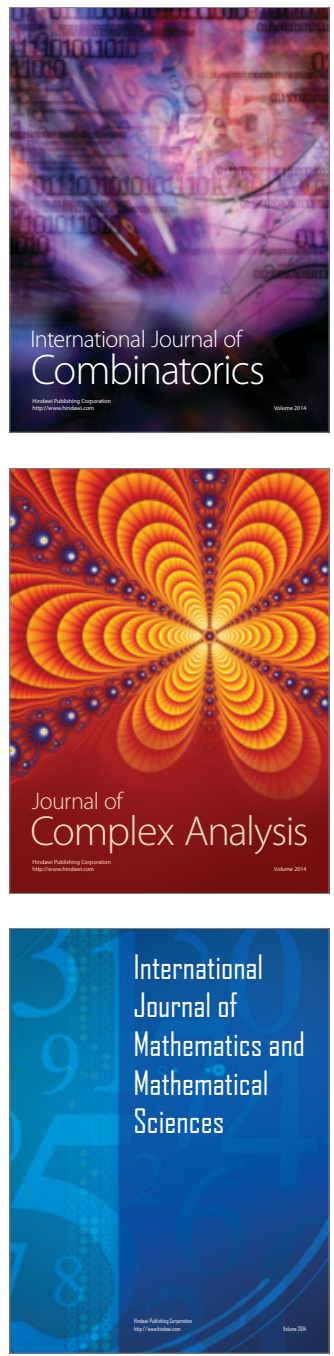
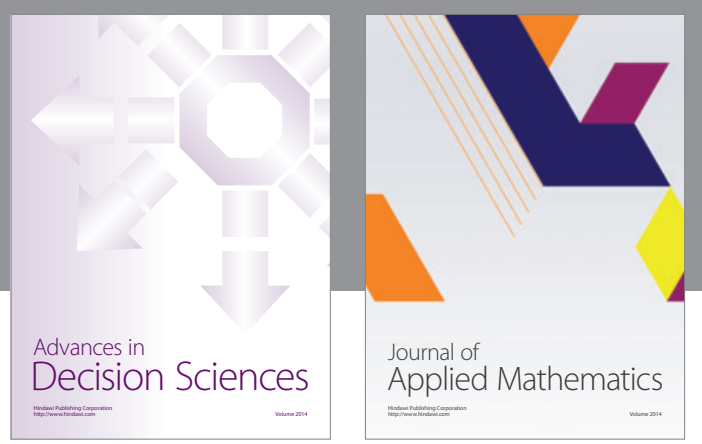

Algebra

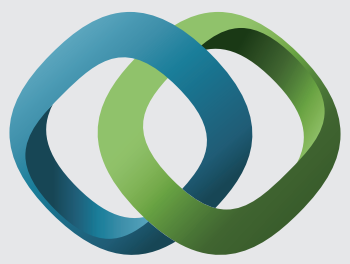

\section{Hindawi}

Submit your manuscripts at

http://www.hindawi.com
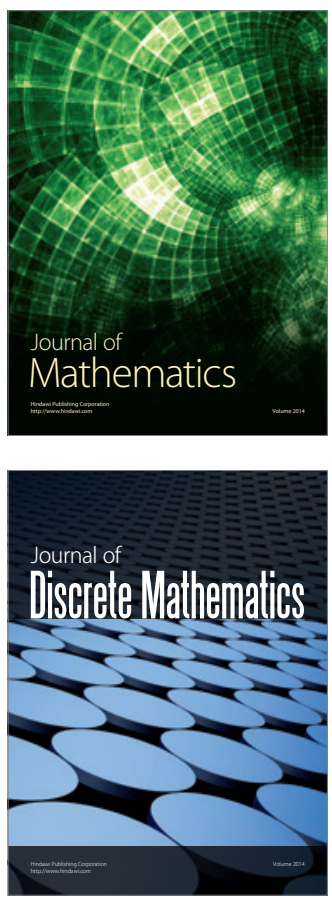

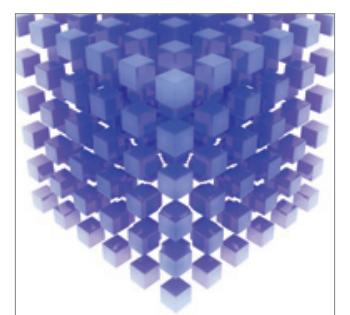

Mathematical Problems in Engineering
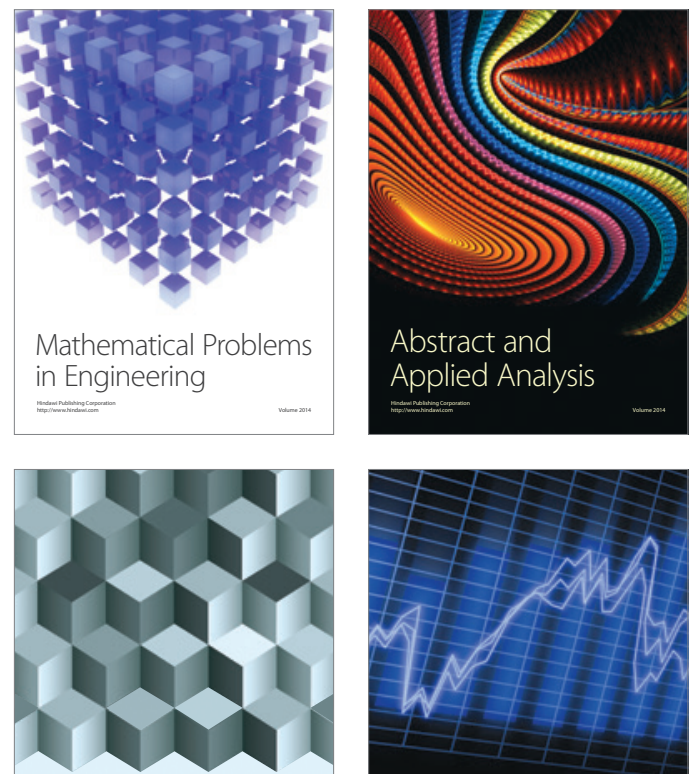

Journal of

Function Spaces

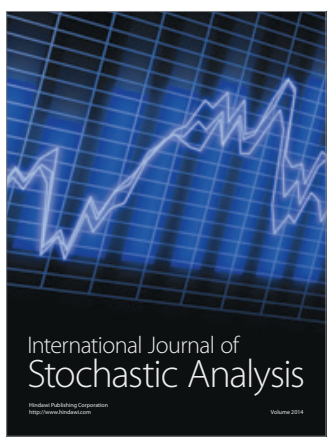

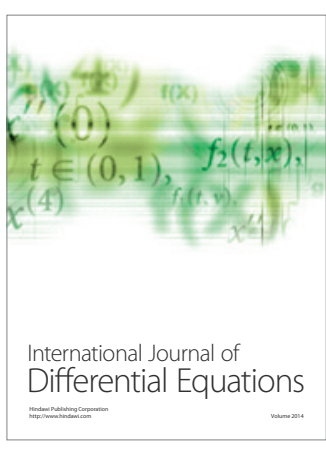
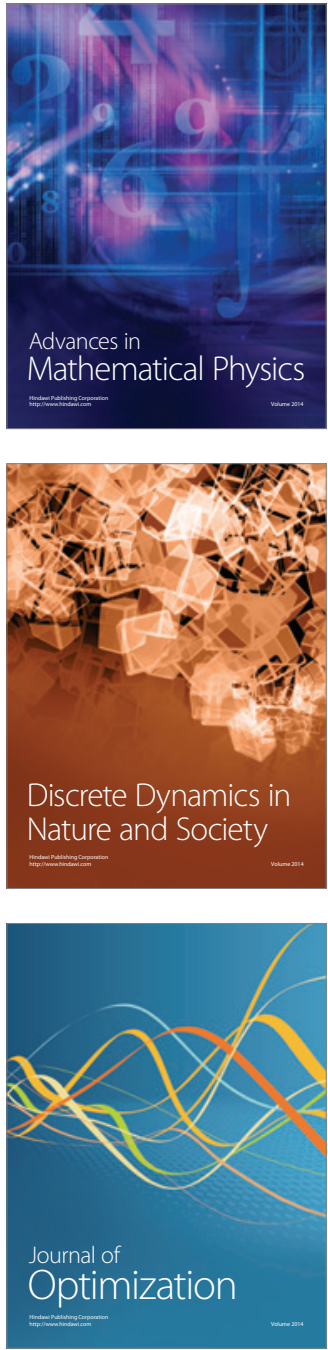